\title{
Effect of Ampelopsis Radix on wound healing in scalded rats
}

Kyungjin Lee, Byonghee Lee, Mi-Hwa Lee, Bumjung Kim, Khanita Suman Chinannai, Inhye Ham and Ho-Young Choi

\begin{abstract}
Background: Ampelopsis Radix has been used as a traditional Korean medicine for the treatment of burns and scalds. However, there has been no scientific research to date on the wound healing properties of Ampelopsis Radix for scald burns. This study aimed to evaluate the healing effect of Ampelopsis japonica root tuber ethanol extract (AJE) on induced cutaneous scald injury in Sprague Dawley (SD) rats.
\end{abstract}

Methods: Hot water scalds were induced in SD rats, who were then divided into the following 5 groups; 1) control group without treatment, 2) positive control group with $1 \%$ Silver sulfadiazine (SSD), 3) Vaseline group, and groups 4) and 5) that used Vaseline containing $5 \%$ and $20 \%$ AJE, respectively. The ointment was applied topically to the experimental rats, once daily for 21 days, starting at $24 \mathrm{~h}$ post induction of the scald injury. Gross examination, measurement of wound size, and histopathological examination were performed. And quantitative measurement of cytokine levels of tumor necrosis factor alpha (TNF-a), interleukin-10 (IL-10), transforming growth factor beta 1 (TGF- $\beta 1$ ), and vascular endothelial growth factor (VEGF) were performed by enzyme-linked immunosorbent assay.

Results: Clinical evaluation showed that the AJE and Vaseline groups, rapidly desquamated scab on day 12 post-scalding; in particular, the $20 \%$ AJE group achieved the greatest extent of skin recovery. Sizes of scald wound were significantly lower on days 12,15, 18, and 21 in the AJE treated groups compared to the control groups. Histopathological evaluation showed a well-organized epithelial layer, angiogenesis, tissue granulation and collagen formation with the exception of inflammatory cells in the AJE-treated groups compared to the control groups on day 14, indicating that tissue regeneration had occurred. AJE treatment decreased TNF-a and increased IL-10 levels on days 2 and 14, indicating the anti-inflammatory action of AJE. The AJE groups also showed a decrease in TGF- $\beta 1$ levels on day 7 and VEGF on day 14 in the serum of scald inflicted SD rat model.

Conclusions: These results suggest that AJE possesses scald wound healing activity via accelerating the scald wound repair during the inflammation and proliferative phases of the healing process.

Keywords: Ampelopsis japonica makino, Scald wound, Wound healing, TNF-a, VEGF

\section{Background}

Recently, health care professionals have been faced with an increasing number of patients suffering from wounds and burns [1]. Chronic, non-healing wounds and their treatment represent a major medical and economic problem [2, 3]. The demand for natural remedies is growing in developing countries because they are safe, cheap and useful to treat burn injury [4-7]. The use of

\footnotetext{
* Correspondence: hychoi@khu.ac.kr

Department of Herbology, College of Korean Medicine, Kyung Hee

University, 26 Kyungheedae-ro, Dongdaemun-gu, Seoul 130-701, Republic of Korea
}

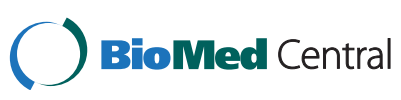

C 2015 Lee et al. This is an Open Access article distributed under the terms of the Creative Commons Attribution License (http://creativecommons.org/licenses/by/4.0), which permits unrestricted use, distribution, and reproduction in any medium, provided the original work is properly credited. The Creative Commons Public Domain Dedication waiver (http:// creativecommons.org/publicdomain/zero/1.0/) applies to the data made available in this article, unless otherwise stated. traditional remedies and plants in treating burns and wounds is an important mode to improve healing, as well as to reduce the financial burdens of treatment. Several plants have been used as a traditional medicine to treat skin disorders and injuries $[4,7-10]$.

Ampelopsis Radix is the dried root tuber of Ampelopsis japonica Makino (Family Vitaceae). The actions of Ampelopsis Radix are to clear heat and eliminate toxins; disperse abscesses and dissipate binds; and to promote wound healing and tissue regeneration. Indications for Ampelopsis Radix are abscesses, cellulitis, carbuncles of the back, deep-rooted boils and sores, scrofula, burns, and scalds 
[11-13]. Recently, external application of Ampelopsis Radix has been reported to have wound healing effect on 80 cases of second degree burn injury in china [14], on scald model of the mice and rats [15], and on sores and ulcers rat model [16]. As mentioned above, Ampelopsis Radix has been used to treat burns and scalds in traditional medicine and reported to have wound healing effects, but no study has yet reported the mechanisms of action of Ampelopsis Radix on burns and scalds.

In the present study, the healing effects and mechanisms of action of the ethanol extract of $A$. japonica Makino root tuber (AJE) on scald wounds using an experimental rat model were investigated.

\section{Methods}

\section{Plant material and extraction}

Dried tuberous root of Ampelopsis japonica Makino (AJ) was purchased from an herbal drug company, DongWooDang Pharmacy Co., Ltd. (Yeongchen, Gyeongsangbuk-do Province, Korea). It was identified by Professor Youngmin Bu. AJ (voucher specimen No. AJ 001) used in this study was deposited in the Laboratory of Herbology, College of Korean Medicine, Kyung Hee University, Seoul, Korea.

AJ (500 g) was extracted three times for $3 \mathrm{~h}$ with $100 \%$ ethanol under heating mantle-reflux. The extract was then condensed with a rotary vacuum evaporator (N-N series, Eyela Co., Japan). The yield of crude extract was $5.12 \%$.

\section{Reagents and equipment}

Veet hair removal cream was purchased from Reckitt Benckiser (France). White Vaseline was purchased from Korea-ione Co. Ltd. (Gyeonggi-Do, Korea). Silmazin 1 \% cream was purchased from Dong-Wha Pharm. Co. Ltd. (Seoul, Korea). Rat interleukin-10 (IL-10) enzyme-linked immunosorbent assay (ELISA) kits and rat transforming growth factor beta 1 (TGF- $\beta 1$ ) ELISA kits were purchased from Cusabio Biotech Co. Ltd. (Wuhan, Hubei Province, China). Rat tumor necrosis factor alpha (TNF$\alpha)$ ELISA kits and rat vascular endothelial growth factor (VEGF) ELISA kits were purchased from Koma Biotech Inc. (Seoul, Korea). The Masson-Goldner trichrome staining kit was purchased from Merck in south Korea (Seoul, Korea), and hematoxylin, eosin Y alcoholic, and Acid Alcohol $\cdot \mathrm{Histo}^{\mathrm{TM}}$ were purchased from BBC Biochemical Co. (USA). Ammonium hydroxide ACS reagent was purchased from Sigma-Aldrich Co. Inc. (USA), and Harris ethyl alcohol and xylene were purchased from J.T.Baker ${ }^{\mathrm{R}}$ (Japan).

In the present study a rotary evaporator (Eyela Co., Japan), ELISA Plate Reader: VersaMax (Molecular Devices Co., USA), digital camera (Sony Corporation, Japan), micro high speed centrifuge (Vision Scientific
Co. Ltd., Korea), HM440E microtome (Carl Zeiss, Germany), Olympus DP70 digital microscope camera and Olympus DP controller software (Olympus Imaging America Inc., USA) were used.

\section{Animals}

To examine the scald-healing effects of AJE, tests were performed on 6-week-old male Sprague-Dawley (SD) rats (weight, 180-220 g; Samtaco, Korea). The rats were housed under controlled conditions $\left(22 \pm 2{ }^{\circ} \mathrm{C}\right.$; lighting, 07:00-19:00) at a pathogen-free animal facility at Kyung Hee University. Food and water were available ad libitum. The experiments were conducted according to the guidelines presented by the Committee for Animal Care and Use of Laboratory Animals, College of Korean Medicine, Kyung Hee University (protocol approval number KHUASP(SE)-13-003).

\section{Scald wound induction and treatment}

To examine the effects of the AJE on the scald wound healing process, scald wounds were induced on the backs of SD rats under anesthesia with ethyl ether. Their dorsal hair was shaved and the residual hair was removed with depilatory cream. After shaving, the dorsal surface was wiped with warm distilled water and $70 \%$ ethanol. The scald wounds were created using the method described by Stevenson et al. [17] with some modification. Hot water induced the second degree scald wound $\left(100{ }^{\circ} \mathrm{C}\right.$ for $\left.10 \mathrm{~s}\right)$ on the dorsal surface in approximate diameter $2.8 \mathrm{~cm}$. After scalding, rats were assigned at random to five groups (Control, Silver sulfadiazine, Vaseline, $5 \%$ and $20 \%$ AJE, $n=8$, respectively) and after $24 \mathrm{~h}, 0.5 \mathrm{~g}$ of the test substance was applied topically to the scald area once daily for 3 weeks as follows: Control (CON), non-treated rats after scald; Silver Sulfadiazine (SSD), wounds treated with reference standard a $1 \%(\mathrm{w} / \mathrm{w})$ SSD cream $(n=8)$; Vaseline, wounds treated Vaseline; $5 \%$ and $20 \%$ AJE, wounds treated with a Vaseline-based 5 and $20 \%$ (w/w) AJE ointment.

\section{Gross examination of the scald wound lesions}

The wounds were grossly examined on day zero following the scald injury, and then at three-day intervals. The lesions were examined using the following criteria: wound bed color, exudates, swelling of the wound surface, and consistency of tissues surrounding the wound. Immediately after inducing the scald wound, the wound area was measured and a wound picture/image was captured using a digital camera. Wound pictures were taken every 3 days and analyzed using ImageJ (Broken Symmetry Software). The percentage wound contracture rate was calculated using following formula: $\%$ Contracture = Specific day wound size / Initial wound size $\times 100$ 


\section{Collection and processing of serum and scald wound tissue}

Blood samples from each group were collected from the tail vein in non-coated tubes, at 2, 7, 14, and 21 days after the scald injury. Collected samples were centrifuged at 8,000 rpm for $40 \mathrm{~min}$, and serum was separated and stored at $-80{ }^{\circ} \mathrm{C}$ until analysis. Scald wounds were excised down to the level of the muscle fascia by sharp dissection and included the surrounding wound margin tissue [18].

\section{Analysis of cytokine and angiogenesis factors}

Serum samples were quantitatively assayed for the inflammatory cytokines TNF- $\alpha$ and IL-10 and the angiogenesis factors TGF- $\beta 1$ and VEGF using ELISA systems. Measurements were performed according to the manufacturer's instructions for TNF- $\alpha$, IL-10, TGF- $\beta 1$, and VEGF using a 96-well microplate reader at $450 \mathrm{~nm}$. All samples for each cytokine were used in triplicate, and optical density measurements were then verified against a standardized curve. The results were averaged and expressed as $\mathrm{pg} / \mathrm{mL}$ or $\mathrm{ng} / \mathrm{mL}$.

\section{Histopathological studies with hematoxylin and eosin and Masson-Goldner trichrome stain}

Rats were sacrificed at days 2, 14, and 21 post-scald using ether, and skin samples were taken for histopathological examination. The skin samples were fixed in $10 \%$ formalin solution. After fixation, the tissues were washed in running tap water, dehydrated in ascending grades of ethyl alcohol, and cleared in xylene. Paraffin embedded tissue sections of $6-\mu \mathrm{m}$ thickness were cut using a microtome and mounted on glass slides. Histological sections were stained with hematoxylin and Eosin (H\&E) and Masson-Goldner trichrome for histological study. Digital photomicrographs were captured at representative locations using a digital camera attached to a microscope. Masson's trichrome stains collagen blue, while cytoplasm, red blood cells, and muscle are stained red; it is typically used to assess the advancement of collagen deposition during the formation of granulation tissue and matrix remodeling [19]. The blue color staining intensity corresponds to the relative quantity of collagen fiber deposited, which reflects the process of synthesis, degradation, and remodeling of tissue [20]. Tissue samples were evaluated for the following histological criteria: extent of re-epithelialization, maturation and organization of the epidermis, granulation tissue formation, collagenization, and inflammatory cells and scar formation in the dermis. The sections were evaluated using a scoring scale of $0-3$ in five categories (epithelialization, vascularization, inflammatory cell response, occurrence of granulation tissue, and collagen deposition), which provided the total graded on a scale of $1-4[21,22]$.

\section{Preliminary phytochemical screening}

Ethanol extract of AJ is usually used in traditional Korean Medicine to treat several skin diseases and several qualitative and quantitative studies were performed using ethanol or methanol extract of AJ [23-27].

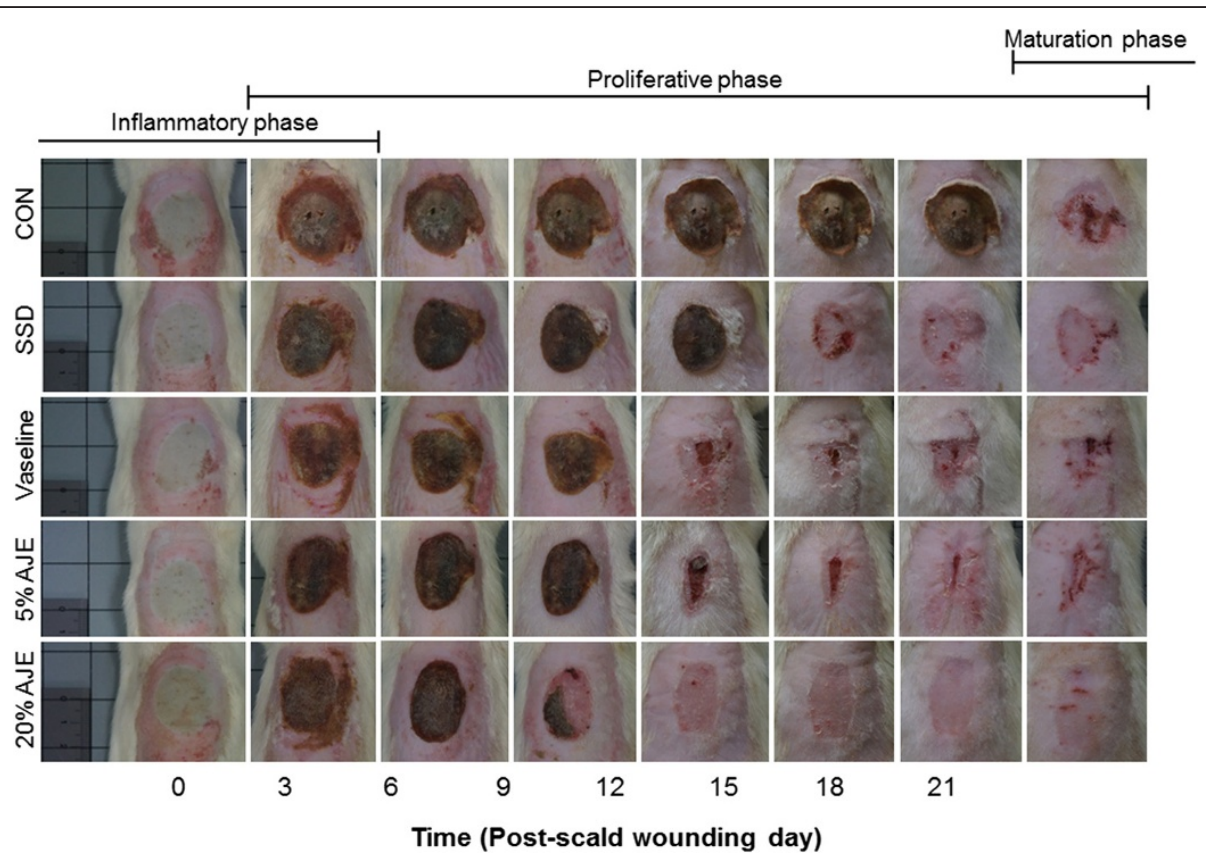

Fig. 1 Gross appearance of the scald wounds. Abbreviations: CON, control; SSD, silver sulfadiazine; AJE, Ampelopsis japonica tuberous root ethanol extract 


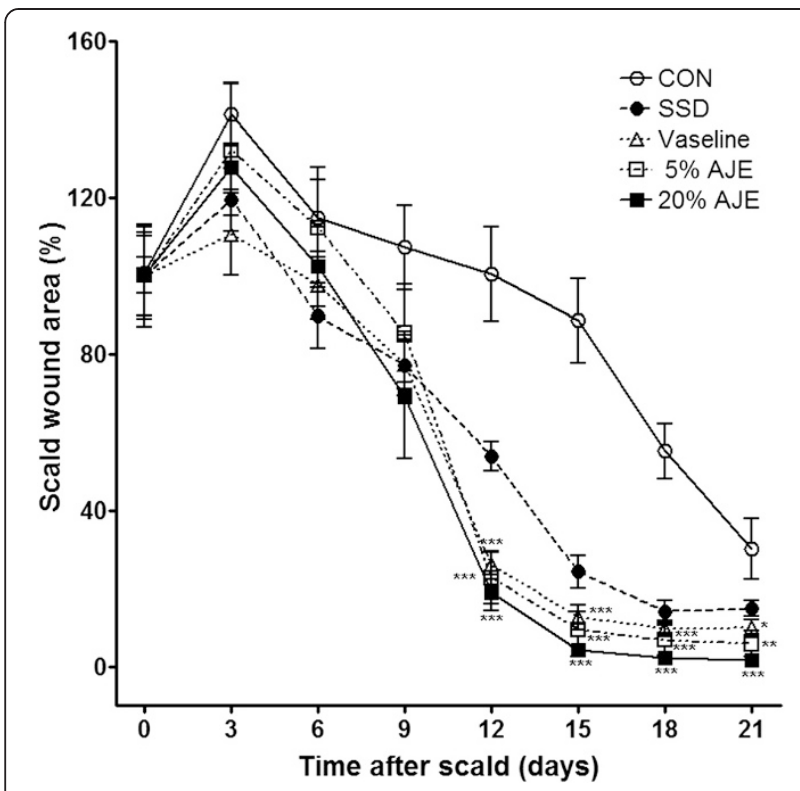

Fig. 2 Changes in scald wound sizes. The percentage wound contracture rate was calculated using following formula: \% Contracture $=$ Specific day wound size / Initial wound size $\times 100$ Abbreviations: CON, control; SSD, silver sulfadiazine; AJE, Ampelopsis japonica tuberous root ethanol extract. Values expressed as mean \pm standard error of the mean $(n=5-8) .{ }^{*} P<0.05,{ }^{* *} P<0.01$, and ${ }^{* * *} P<0.001$ vs. control

\section{Statistical analysis}

Data are expressed as mean \pm standard error of the mean (SEM). All the statistical comparisons were made using one-way analysis of variance (ANOVA) followed by the Tukey's post-hoc test with SPSS v.13.0 statistical analysis software (SPSS Inc., USA). P values less than 0.05 were considered statistically significant.

\section{Results and discussion}

\section{Gross examination}

Observations of any changes in the overall appearance of the wounds took place on days $0,3,6,9,12$, 15, 18, and 21. From day 3 to day 9, the ointmenttreated groups exhibited yellowish-brown, moist, soft, and supple scabs with red rims along the margins. After day 12, the Vaseline and AJE treated group did not exhibit thick scabs and bleeding, whereas the control and SSD treated group showed thick, dry, and dark brown scabs that were intact. By day 15, re-epithelialization was observed in all ointmenttreated groups but the control group. Wounds healed best in the $20 \%$ CGE treated group after day 12: the wounds nearly healed, while the wound of control and SSD-treated group still exhibited a dry appearance with dark brown scab (Fig. 1).

\section{Measurement of scald wound size}

In the first 0 to 9 days, there was nearly no difference between ointment-treated groups and control group in the wound contraction ratio. On day 12, the healing rates in all ointment-treated groups were significantly higher than that of the control group. On day 12, the $20 \%$ AJE group showed the best healing rate with a wound size of $19.0 \pm 4.6 \%$. AJE groups showed the lowest mean size of wound area from day 12 to day 21 (Fig. 2).

\section{Histopathological examination}

Histopathological severity evaluation revealed that all of the scald wounds were in the deep second degree category. 1) Epithelialization: Epidermal regeneration was not observed on day 2 . On day 14, the occurrence of epithelialization (epithelialization score) was better than the day 2. The score was significantly greater in AJE treated group lesions than in control group. Epithelialization was observed rapidly ongoing in all groups except

Table 1 Comparison of histopathological scores among the groups

\begin{tabular}{|c|c|c|c|c|c|}
\hline \multirow[t]{2}{*}{ Day } & \multicolumn{5}{|c|}{ Epithelialization } \\
\hline & $\mathrm{CON}$ & SSD & Vaseline & $5 \%$ AJE & $20 \%$ AJE \\
\hline 2 & $0.0 \pm 0.0$ & $0.0 \pm 0.0$ & $0.3 \pm 0.3$ & $0.00 \pm 0.00$ & $0.1 \pm 0.1$ \\
\hline 14 & $1.5 \pm 0.4$ & $2.3 \pm 0.07$ & $2.9 \pm 0.1^{* *}$ & $2.9 \pm 0.1^{* * *}$ & $3.0 \pm 0.0^{* *}$ \\
\hline \multirow[t]{2}{*}{21} & $1.0 \pm 0.0$ & $2.7 \pm 0.18^{* * *}$ & $2.9 \pm 0.1^{* * *}$ & $2.9 \pm 0.1^{* * *}$ & $3.0 \pm 0.0^{* * *}$ \\
\hline & \multicolumn{5}{|c|}{ Vascularization } \\
\hline 2 & $0.0 \pm 0.0$ & $0.0 \pm 0.0^{\#}$ & $1.6 \pm 0.6^{*}$ & $1.8 \pm 0.3^{*}$ & $1.6 \pm 0.4^{*}$ \\
\hline 14 & $1.2 \pm 0.4$ & $1.7 \pm 0.4$ & $1.9 \pm 0.1$ & $2.2 \pm 0.2$ & $2.2 \pm 0.2$ \\
\hline \multirow[t]{2}{*}{21} & $1.5 \pm 0.2$ & $1.7 \pm 0.1$ & $1.9 \pm 0.2$ & $2.3 \pm 0.3$ & $2.5 \pm 0.1^{*}$ \\
\hline & \multicolumn{5}{|c|}{ Granulation } \\
\hline 2 & $0.0 \pm 0.0$ & $0.6 \pm 0.1^{*}$ & $1.2 \pm 0.0^{* * *}$ & $1.5 \pm 0.0^{* * *}$ & $1.3 \pm 0.1^{* * *}$ \\
\hline 14 & $1.4 \pm 0.2$ & $1.9 \pm 0.1$ & $2.0 \pm 0.1^{*}$ & $2.6 \pm 0.1^{* * *, \#}$ & $2.8 \pm 0.2^{* * *, \#}$ \\
\hline \multirow[t]{2}{*}{21} & $2.8 \pm 0.0$ & $2.8 \pm 0.1$ & $2.9 \pm 0.1$ & $2.9 \pm 0.1$ & $3.0 \pm 0.1$ \\
\hline & \multicolumn{5}{|c|}{ Inflammation } \\
\hline 2 & $2.6 \pm 0.2$ & $2.0 \pm 0.1$ & $2.9 \pm 0.1$ & $2.8 \pm 0.2$ & $2.7 \pm 0.1$ \\
\hline 14 & $2.9 \pm 0.1$ & $2.3 \pm 0.2$ & $2.3 \pm 0.1^{*}$ & $1.3 \pm 0.2^{* * * * \# \# \#}$ & $1.2 \pm 0.1^{* * *}, \# \#$ \\
\hline \multirow[t]{2}{*}{21} & $2.3 \pm 0.3$ & $1.3 \pm 0.1^{*}$ & $1.3 \pm 0.1^{*}$ & $0.9 \pm 0.1^{* *}$ & $0.9 \pm 0.3^{* *}$ \\
\hline & \multicolumn{5}{|c|}{ Collagen deposition } \\
\hline 2 & $1.2 \pm 0.1$ & $1.1 \pm 0.2$ & $1.5 \pm 0.1$ & $1.3 \pm 0.2$ & $1.1 \pm 0.2$ \\
\hline 14 & $2.2 \pm 0.2$ & $2.5 \pm 0.2$ & $2.7 \pm 0.1$ & $2.8 \pm 0.1^{*}$ & $2.9 \pm 0.1^{* *}$ \\
\hline \multirow[t]{2}{*}{21} & $2.3 \pm 0.2$ & $2.6 \pm 0.1$ & $2.7 \pm 0.2$ & $2.9 \pm 0.1^{*}$ & $3.0 \pm 0.1^{*}$ \\
\hline & \multicolumn{5}{|c|}{ Total score } \\
\hline 2 & $1.0 \pm 0.0$ & $1.4 \pm 0.1$ & $1.2 \pm 0.0$ & $1.4 \pm 0.1$ & $1.5 \pm 0.2$ \\
\hline 14 & $2.1 \pm 0.1$ & $2.5 \pm 0.1$ & $2.7 \pm 0.1^{* *}$ & $3.3 \pm 0.2^{* * *}, \#$ & $3.0 \pm 0.1^{* * *}$ \\
\hline 21 & $2.4 \pm 0.0$ & $3.2 \pm 0.1^{* *}$ & $3.3 \pm 0.1^{* * *}$ & $3.6 \pm 0.1^{* * *}$ & $3.9 \pm 0.1^{* * *,}$, \# \\
\hline
\end{tabular}

Abbreviations: CON control, SSD silver sulfadiazine, AJE Ampelopsis japonica tuberous root ethanol extract. Values are expressed as mean \pm standard error of the mean $(n=3-8) .{ }^{*} P<0.05,{ }^{* *} P<0.01,{ }^{* * *} P<0.001$ vs. Control, ${ }^{\#} P<0.05$, ${ }^{\# \#} P<0.01,{ }^{\# \#} P<0.001$ vs. Vaseline 


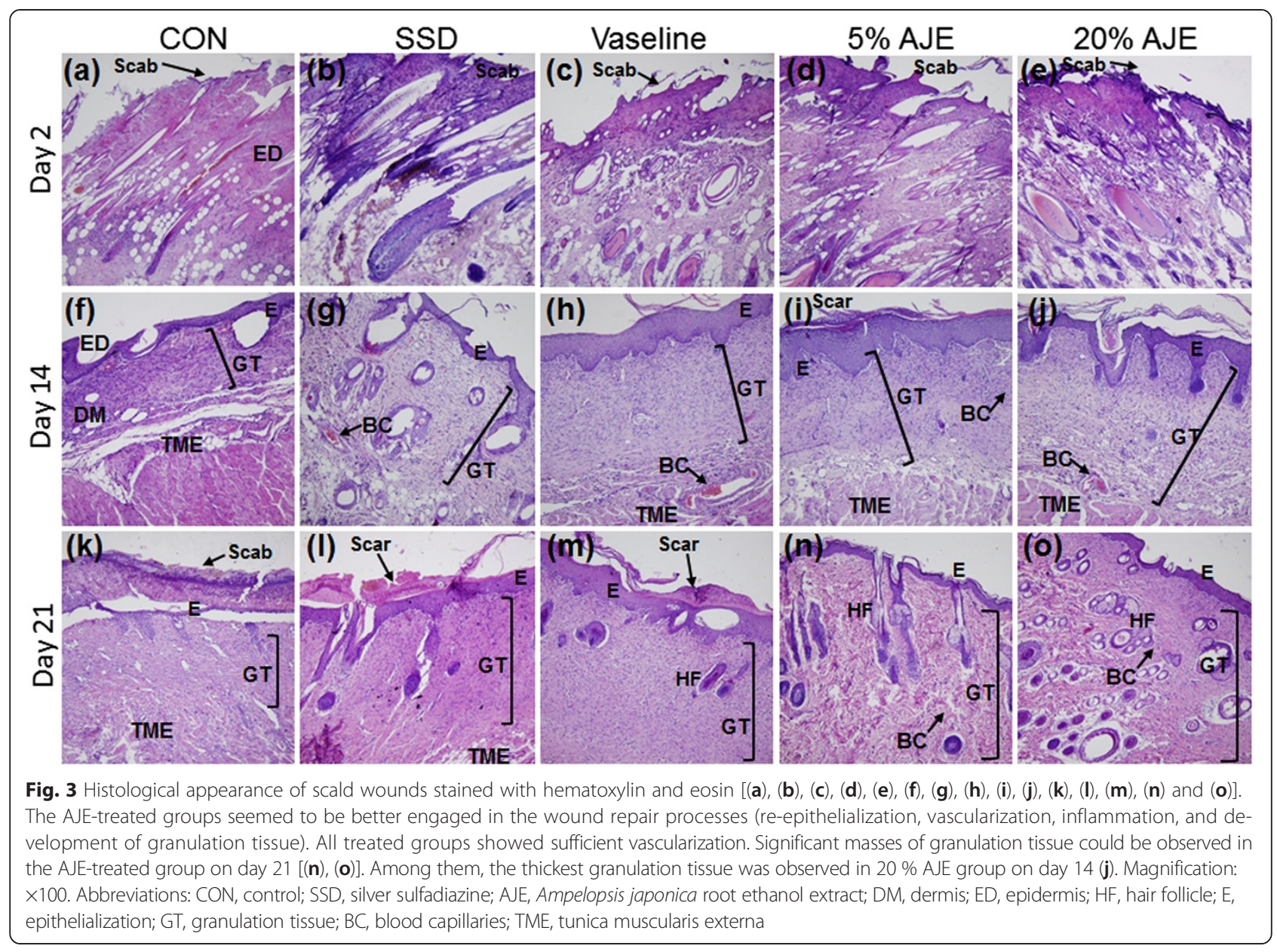

for the control group. On day 14, 21, epithelialization scores were significantly higher in $5 \%$ and $20 \%$ AJE treated group than in the control group. 2) Vascularization: Vascularization was already occurred in Vaseline and AJE treated groups on day 2. On the other hand, vascularization did not yet occurred in control and SSD treated group on day 2 . On day 14, vascularization scores were increased in all groups. There were increased dense blood vessels distributed deeply in the tissue. On day 21, vascularization score was highest in AJE treated groups and increased slightly more on day 14.3) Granulation: On day 2, in all scald wound injured groups, there were damage of epidermis, dermis and subcutaneous tissue were observed in the scalded region. The granulation tissue obtained from topically treated rats showed an increase in number of fibroblasts. On day 14, a little-advanced organization of granulation tissue has begun forming in the dermis. Among them, $20 \%$ AJE group was formed most thick of the granulation tissue area. In contrast, the control group had observed irregularly forming granulation tissue. On day 21 , the most significant increase in the granulation tissue was observed in ointment treated groups than control group. 4) Inflammation: On day 2, inflammatory cell infiltration was significantly increased in the all groups. On day 14 , the control group exhibited wide area of ulcerations containing inflammatory cell, mild degrees of inflammation. $5 \%$ AJE and $20 \%$ AJE treated group showed mild inflammation. On day 21, the inflammatory cells were decreased in ointment treated groups. 5) Collagen deposition: On day 2, the collagen deposition was observed in all groups. On day 14, new formed collagen appeared abundant in the dermis. On day 21, the collagen was observed in the dermis in AJE groups, while collagen fibers filled the dermis in the control group. Collagen regeneration was significantly increased in the AJE groups at on day 14 and 21. 6) Total histopathology score: Total histopathological evaluation scores in ointment treated groups were significantly better than control group. Further, total histopathological evaluation scores in the AJE groups were significantly better than Vaseline group (Table 1, Figs. 3 and 4).

\section{Quantification of TNF- $\alpha$ and IL-10}

TNF- $\alpha$, a pro-inflammatory cytokine, is up-regulated during the inflammatory phase of wound healing [28] and appears to be involved in initiating the early wound healing response [29]. Low levels of TNF- $\alpha$ can promote wound 


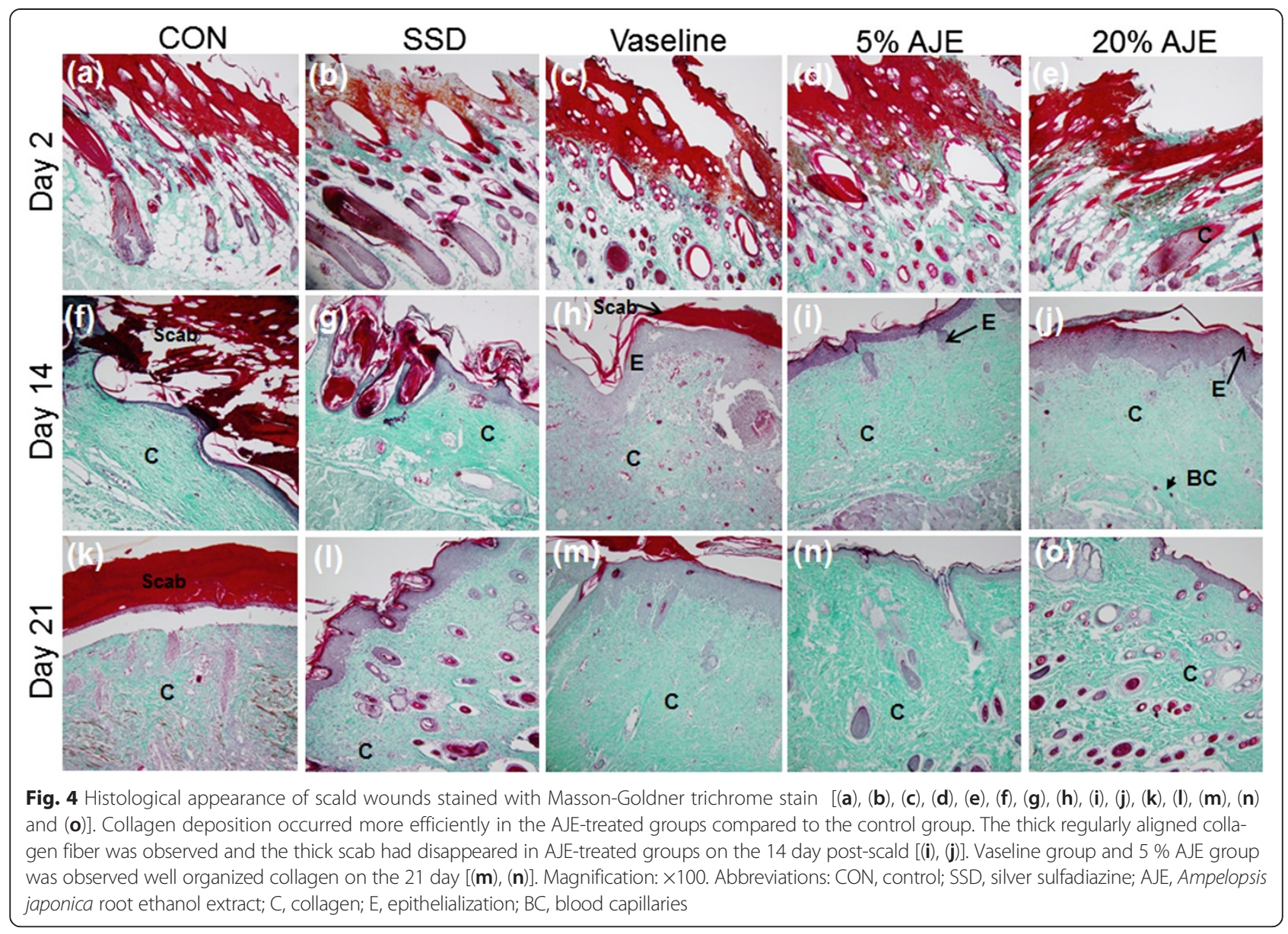

healing indirectly but high levels of TNF- $\alpha$ can delay wound healing [28]. Therefore up-regulated TNF- $\alpha$ during the inflammatory phase should decreased for the rapid wound healing. IL-10 is an anti-inflammatory cytokine produced by various cells, including macrophages and Tlymphocytes, and is also involved in angiogenesis [30]. It is known to be a major regulator in suppressing the inflammatory response. IL-10 inhibits the synthesis of proinflammatory cytokines such as IL-1B, IL- 6 and TNF- $\alpha$ in activated macrophages [31]. In the present study, serum samples were collected on days 2 and 14 to determine the impact of the scald wound on the pro-inflammatory response. On day 2, the levels of TNF- $\alpha$ were higher in the $5 \%$ and $20 \%$ AJE treated group compared to the control group and Vaseline treated group. On day 14, TNF- $\alpha$ level was decreased in all groups and TNF- $\alpha$ level was lower in the $20 \%$ AJE treated group than Vaseline treated group (Fig. 5). On the other hand, the IL-10 levels were higher on day 14 in AJE treated groups than in the control group and Vaseline treated group. On day 21, all experimental groups were maintained higher levels of IL-10 than the control group throughout the proliferative phase (Fig. 6). In addition, the histopathological sections of the dorsal skin on day 14 in the post-scald AJE treatment groups

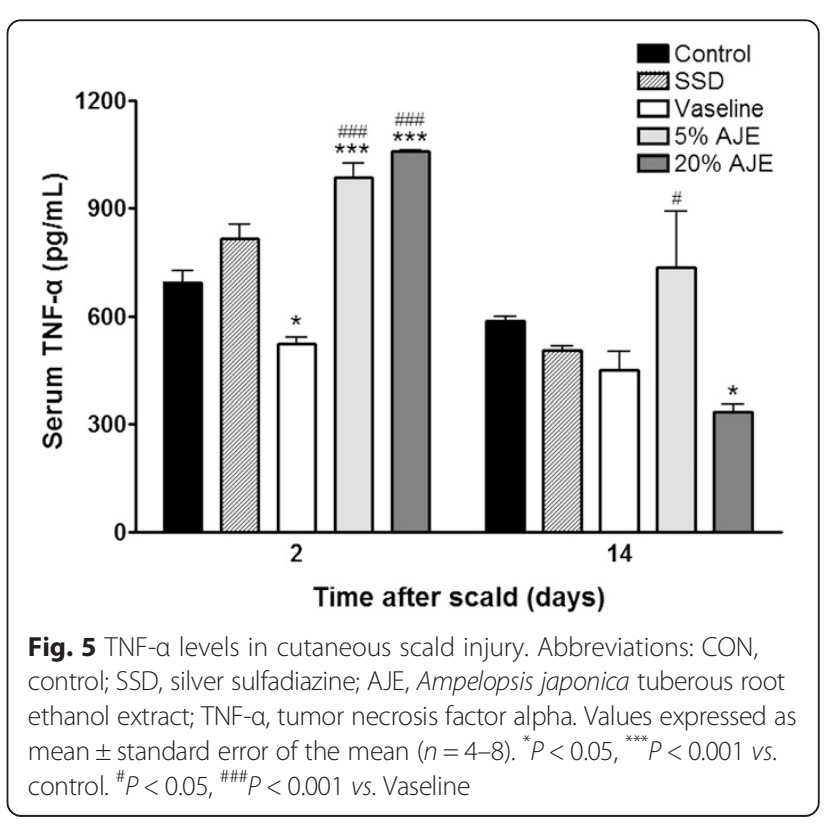




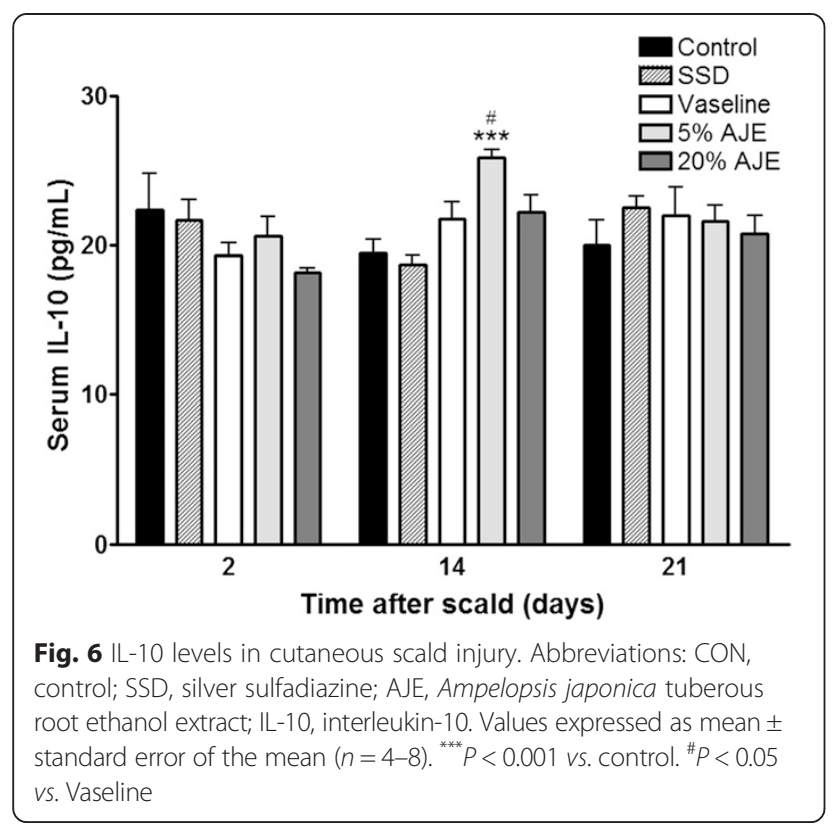

showed a lower number of inflammatory cells than the control group (Table 1). These result suggested that AJE can reduce wound size and promote wound healing via decreasing of up-regulated TNF- $\alpha$ levels and Increasing of IL-10.

\section{Quantification of TGF- $\beta 1$}

TGF- $\beta 1$ is a key growth factor secreted by several cells and is involved in a number of processes in wound healing, i.e., inflammation, angiogenesis, fibroblast proliferation, collagen synthesis, and remodeling of new extracellular matrix $[32,33]$. Increased production of TGF- $\beta 1$ supports faster

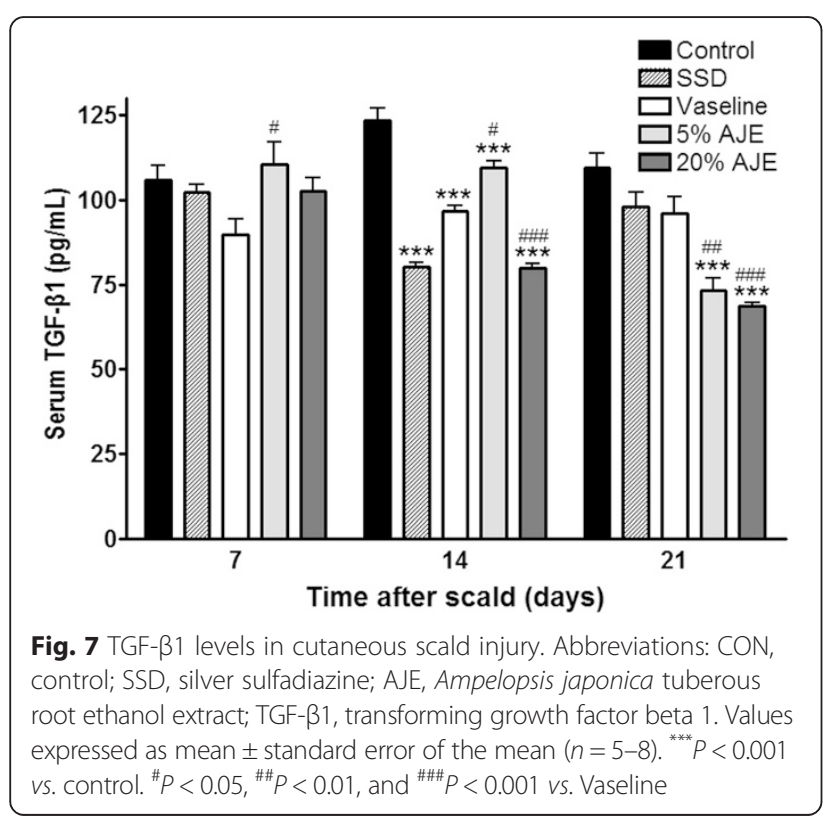

re-epithelialization but hypertrophic scarring and keloid formation are occurred by over-expression of TGF- $\beta 1$ during the late stages of wound healing [34]. Therefore, TGF$\beta 1$ should decreased after re-epithelialization for the wound healing without scar. In the present study, the levels of TGF- $\beta 1$ were increased in all groups and decreased after 7 to 21 days in the AJE treated groups. On day 14, TGF- $\beta 1$ levels in all experimental groups were lower than control group. Further, TGF- $\beta 1$ level in $20 \%$ AJE treated group was lower than Vaseline treated group. On day 21, TNF- $\beta 1$ levels were significantly lower in AJE-treated groups than control group and Vaseline treated group (Fig. 7). These results supported that the scabs were removed from the AJE treated groups, but the scabs in the control group were still present on histopathological observation and general evaluation (Figs. 1, 2, and 3). Additionally, the ability of TGF- $\beta 1$ to stimulate collagen production is so potent that it can result in significant changes in histopathology on AJEtreatment.

\section{Quantification of VEGF}

Angiogenesis is an important factor in proliferative phase of wound healing. VEGF is one of the most potent proangiogenic growth factors in the skin [35]. In the last stage of wound healing, VEGF plays a role of promoting scar formation [36]. In the present study, AJE treated groups showed higher levels of VEGF than the other groups on day 7. On 14 day, the VEGF levels of control, SSD, and Vaseline treated groups were increased. On the other hand, VEGF levels of AJE treated groups were decreased. On day 21, all experimental groups were decreased in production of VEGF. Especially, VEGF levels of AJE treated groups were lower than the other groups (Fig. 8). In

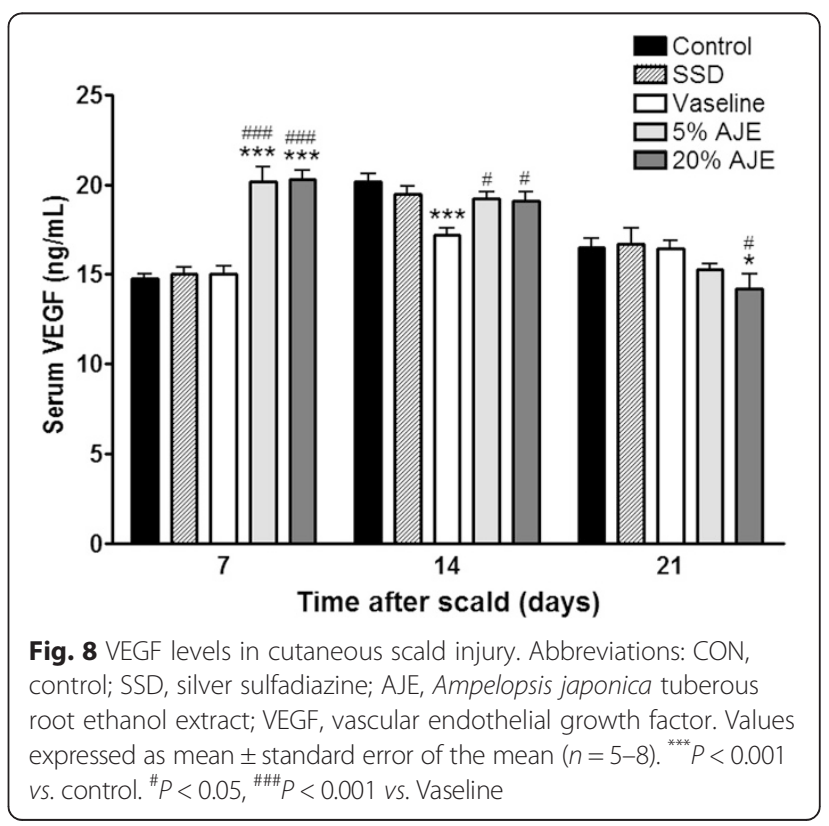


addition, after histopathological scoring with H\&E staining, the AJE treated groups showed an increase in re-epithelialization and neovascularization compared to the Vaseline treated group and the deposition of collagen in the AJE treated group was greater than in the other groups on day 14 (Figs. 3 and 4). These results suggested that AJE could heal scald wounds faster and result in less scarring than other treatments by regulating VEGF in the whole wound healing process.

\section{Preliminary phytochemical screening}

Preliminary phytochemical screening of AJE showed the presence of catechin, epicatechin, resveratrol, schizandriside, gallocatechin, and epicatechin gallate [24, 25, 37]. Catechin and resveratrol are the main costituents of AJE [24] and catechin was present at high concentrations in AJE [37]. Catechin [38, 39] and resveratrol $[23,40,41]$ are well known anti-inflammatory and wound-healing compounds. Additionally, epicatechin, epicatechin gallate, and gallocatechin [39, 42, 43] are reported to have wound-healing properties. Therefore, the beneficial effects of AJE might be mainly attributable to catechin and resveratrol; other known and unknown compounds in AJE also might contribute to its effects.

\section{Conclusions}

In conclusion, AJE showed faster and more effective wound healing activities than SSD and Vaseline in the skin of experimentally scalded rats. Histopathological evaluation results showed better re-epithelialization, vascularization, granulation tissue formation, and collagen deposition in the AJE treated groups than the other groups. These effects were due to the appropriate regulation of TNF- $\alpha$, IL-10, TGF- $\beta 1$, and VEGF. AJE could be of beneficial use in wound healing of scald injury.

\footnotetext{
Abbreviations

AJE: A. japonica root tuber ethanol extract; SSD: $1 \%$ Silver sulfadiazine; TNF-a: Tumor necrosis factor alpha; IL-10: Interleukin-10; TGF- 31 : Transforming growth factor beta 1; VEGF: Vascular endothelial growth factor; AJ: Dried tuberous root of Ampelopsis japonica Makino.
}

\section{Competing interests}

The authors declare that they have no competing interests.

\section{Authors' contributions}

$\mathrm{ML}, \mathrm{KL}, \mathrm{BK}, \mathrm{KC}$ and $\mathrm{IH}$ performed the animal experiments. $\mathrm{KL}$ and $\mathrm{BL}$ participated in the writing of the manuscript. $\mathrm{HC}$ and $\mathrm{BL}$ conceived the study and participated in its design and coordination. $\mathrm{HC}$ helped draft the manuscript. All authors read and approved the final manuscript.

\section{Acknowledgements}

This study was supported by a grant from the High Value-added Food Technology Development Program, Korea Institute of Planning \& Evolution for Technology in Food, Agriculture, Forestry \& Fisheries (314071-03-1-HD020).
Received: 27 February 2015 Accepted: 29 June 2015

Published online: 08 July 2015

\section{References}

1. Mogosanu GD, Grumezescu AM. Natural and synthetic polymers for wounds and burns dressing. Int J Pharm. 2014;463(2):127-36.

2. Lay-flurrie K. Honey in wound care: effects, clinical application and patient benefit. Br J Nurs. 2008;17(11):S30. S32-36.

3. Riedel K, Ryssel H, Koellensperger E, Germann G, Kremer T. Pathogenesis of chronic wounds. Chirurg. 2008;79(6):526-34.

4. Narayan S, Sasmal D, Mazumder PM. Evaluation of the wound healing effect of herbal ointment formulated with Salvia splendens (scarlet sage). Int J Pharm Pharm Sci. 2011;3(3):195-9.

5. Abdel-Azim NS, Shams KA, Shahat A, El Missiry MM, Ismail SI, Hammouda FM. Egyptian herbal drug industry: challenges and future prospects. Res J Med Plant. 2011;5:136-44.

6. Sharp A. Beneficial effects of honey dressings in wound management. Nurs Stand. 2009;24(7):66-8. 70, 72 passim.

7. Albertyn R, Berg A, Numanoglu A, Rode H. Traditional burn care in subSaharan Africa: a long history with wide acceptance. Burns. 2015;41(2):203-11.

8. Abdulla MA, Ahmed KA, Ali HM, Noor SM, Ismail S. Wound healing activities of Rafflesia hasseltii extract in rats. J Clin Biochem Nutr. 2009;45(3):304-8.

9. Lodhi S, Pawar RS, Jain AP, Singhai AK. Wound healing potential of Tephrosia purpurea (Linn.) Pers. in rats. J Ethnormacol. 2006;108(2):204-10.

10. Tan Y, Wang KY, Wang N, Li G, Liu D. Ectopic expression of human acidic fibroblast growth factor 1 in the medicinal plant, Salvia miltiorrhiza, accelerates the healing of burn wounds. BMC Biotechnol. 2014;14:74.

11. State Pharmacopoeia Committee. Pharmacopoeia of the People's Republic of China. Beijing: People's Medical Publishing House; 2010.

12. Zhonghua Bencao Edit Committee. Zhonghua Bencao. Shanghai: Shanghai Science and Technology Publications; 1999.

13. Li HJ, Zhang L, Wang SG. Pharmacological action and clinical application of Radix Ampelopsis. Food Drug. 2007;10:60-2.

14. Chen SM, Ye JX, Ye WH, Lu SJ, Tan ZL. Treatment of $\|^{\circ}$ burn by Chuangmian Ling: A clinical observation of 80 cases. Xin Zhong Yi. 2003;35(10):22-3.

15. Miao J, Bai M, Guo X, Miao M. Ampelopsis on the scald model of the mice and rats. Pharmacol Clin Chinese Mater Med. 2012;28(4):65-8.

16. Tang PP, Guo XF, Bai M, Mial MS. Effect of Ampelopsis external application on sores ulcers model. China J Tradit Chin Med Pharm. 2012;27(3):702-5.

17. Stevenson JM, Gamelli RL, Shankar R. A mouse model of burn wounding and sepsis. Methods Mol Med. 2003;78:95-105.

18. Frank S, Kampfer H. Excisional wound healing. An experimental approach. Methods Mol Med. 2003;78:3-15.

19. Braiman-Wiksman $L$, Solomonik I, Spira $R$, Tennenbaum $T$. Novel insights into wound healing sequence of events. Toxicol Pathol. 2007;35(6):767-79.

20. Aramwit $P$, Sangcakul A. The effects of sericin cream on wound healing in rats. Biosci Biotechnol Biochem. 2007;71(10):2473-7.

21. Karadag CA, Birtane M, Aygit AC, Uzunca K, Doganay L. The efficacy of linear polarized polychromatic light on burn wound healing: an experimental study on rats. J Burn Care Res. 2007;28(2):291-8.

22. Peppa M, Brem H, Ehrlich P, Zhang JG, Cai W, Li Z, et al. Adverse effects of dietary glycotoxins on wound healing in genetically diabetic mice. Diabetes. 2003;52(11):2805-13.

23. Sen CK, Khanna S, Gordillo G, Bagchi D, Bagchi M, Roy S. Oxygen, oxidants, and antioxidants in wound healing: an emerging paradigm. Ann N Y Acad Sci. 2002;957:239-49.

24. Nho KJ, Chun JM, Kim DS, Kim HK. Ampelopsis japonica ethanol extract suppresses migration and invasion in human MDAMB231 breast cancer cells. Mol Med Rep. 2015;11(5):3722-8.

25. Kim IH, Umezawa M, Kawahara N, Goda Y. The constituents of the roots of Ampelopsis japonica. J Nat Med. 2007;61(2):224-5.

26. He J, Xian J, Song YY, Song SJ. Chemical constituents of the root of Ampelopsis japonica (Thunb.) Makino. J Shenyang Pharmaceutical University. 2008;25(8):636-8.

27. Libin G, Yan L, Shuipin C. Study on the chemical constituents of Ampelopsis Japonica (Thunb.) Makino. Acad J Guangdong Coll Pharm. 1996;12(3):145-7.

28. Werner $\mathrm{S}$, Grose R. Regulation of wound healing by growth factors and cytokines. Physiol Rev. 2003;83(3):835-70. 
29. Mohan RR, Kim WJ, Wilson SE. Modulation of TNF-alpha-induced apoptosis in corneal fibroblasts by transcription factor NF-kappaB. Invest Ophthalmol Vis Sci. 2000;41(6):1327-36.

30. Silvestre JS, Mallat Z, Duriez M, Tamarat R, Bureau MF, Scherman D, et al. Antiangiogenic effect of interleukin-10 in ischemia-induced angiogenesis in mice hindlimb. Circ Res. 2000;87(6):448-52

31. Sato Y, Ohshima T, Kondo T. Regulatory role of endogenous interleukin-10 in cutaneous inflammatory response of murine wound healing. Biochem Biophys Res Commun. 1999;265(1):194-9.

32. Roberts AB, Sporn MB, Assoian RK, Smith JM, Roche NS, Wakefield LM, et al. Transforming growth factor type beta: rapid induction of fibrosis and angiogenesis in vivo and stimulation of collagen formation in vitro. Proc Natl Acad Sci U S A. 1986;83(12):4167-71.

33. Nall AV, Brownlee RE, Colvin CP, Schultz G, Fein D, Cassisi NJ, et al. Transforming growth factor beta 1 improves wound healing and random flap survival in normal and irradiated rats. Arch Otolaryngol Head Neck Surg. 1996;122(2):171-7.

34. Pakyari M, Farrokhi A, Maharlooei MK, Ghahary A. Critical role of transforming growth factor beta in different phases of wound healing. Adv Wound Care. 2013;2(5):215-24.

35. Johnson KE, Wilgus TA. Vascular endothelial growth factor and angiogenesis in the regulation of cutaneous wound repair. Adv Wound Care. 2014;3(10):647-61.

36. Nissen NN, Polverini PJ, Gamelli RL, DiPietro LA. Basic fibroblast growth factor mediates angiogenic activity in early surgical wounds. Surgery. 1996;119(4):457-65.

37. Park H, Shim JS, Kim HG, Lee H, Oh MS. Ampelopsis Radix protects dopaminergic neurons against 1-methyl-4-phenylpyridinium/1-methyl-4phenyl-1,2,3,6-tetrahydropyridine-induced toxicity in Parkinson's disease models in vitro and in vivo. Evid Based Complement Alternat Med. 2013; Article ID:346438.

38. Braganca de Moraes CM, Melo DA, Santos RC, Bitencourt S, Mesquita FC, dos Santos de Oliveira F, et al. Antiproliferative effect of catechin in GRX cells. Biochem Cell Biol. 2012;90(4):575-84.

39. Schmidt CA, Murillo R, Bruhn T, Bringmann G, Goettert M, Heinzmann B, et al. Catechin derivatives from Parapiptadenia rigida with in vitro woundhealing properties. J Nat Prod. 2010;73(12):2035-41.

40. Yaman I, Derici H, Kara C, Kamer E, Diniz G, Ortac R, et al. Effects of resveratrol on incisional wound healing in rats. Surg Today. 2013;43(12):1433-8.

41. Brakenhielm E, Cao R, Cao Y. Suppression of angiogenesis, tumor growth, and wound healing by resveratrol, a natural compound in red wine and grapes. FASEB J. 2001;15(10):1798-800.

42. Kapoor M, Howard R, Hall I, Appleton I. Effects of epicatechin gallate on wound healing and scar formation in a full thickness incisional wound healing model in rats. Am J Pathol. 2004;165(1):299-307.

43. McKelvey KJ, Appleton I. Epicatechin gallate improves healing and reduces scar formation of incisional wounds in type 2 diabetes mellitus rat model. Wounds. 2012;24(3):55-7.

\section{Submit your next manuscript to BioMed Central and take full advantage of:}

- Convenient online submission

- Thorough peer review

- No space constraints or color figure charges

- Immediate publication on acceptance

- Inclusion in PubMed, CAS, Scopus and Google Scholar

- Research which is freely available for redistribution 